\title{
Management of Infected Mesh Following Ventral Hernia Repair
}

\author{
Osama Abbas El Kattan, Ahmed Abd El Mawgod Abd El Salam, Ahmed El Sayed Fathy \\ Mohamed \\ Department of General Surgery, Faculty of Medicine, Al-Azhar University \\ Corresponding author: Ahmed El Sayed Fathy Mohamed, Mobile: 01142434949, email: dr.aesaleh@ gmail.com
}

\begin{abstract}
Background: abdominal hernia represents a major health care burden. With over 350,000 repairs performed annually in the United States, millions of dollars are consumed with results that are often far from ideal. The use of the prosthesis in the abdominal wall hernia repair (AWHR) has introduced new problems. Although mesh has reduced hernia recurrence rates, it has its own set of complications. So, mesh infection is one of the most devastating complications after the implantation of any mesh. Objective: this work aimed to focus on management of infected mesh after ventral hernia repair. Patients and Methods: this study was conducted in the Department of Surgery, Faculty of Medicine, Al-Azhar University Hospitals from September 2016 until March 2018. The study included 40 patients with surgical mesh infections after the repair of the ventral hernia. Results: cases with laparoscopic hernia repair, minor infections and a patient unfit for surgery were excluded for any medical reason. And after taking the history of the disease and clinical examination and the necessary investigations and the most important is to take a sample of infected fluid over the mesh to determine the type of infection caused by this or doing fistulogram if the fistula connected to the intestine small or large. Conclusion: research of best practices in surgical technique, preoperative care and mesh materials is ongoing, and much remains to be learned on prevention and management of this complex and potentially devastating complication.
\end{abstract}

Keywords: abdominal wall hernia repair, partial removal of mesh, Complete mesh removal

\section{INTRODUCTION}

Abdominal hernia represents a major health care burden. With over 350,000 repairs performed annually in the United States, millions of dollars are consumed with results that are often far from ideal ${ }^{(1)}$. The use of the prosthesis in the abdominal wall hernia repair (AWHR) has introduced new problems. Although mesh has reduced hernia recurrence rates, it has its own set of complications. So, mesh infection is one of the most devastating complications after the implantation of any mesh (2). The risk of infection in AWHR appeared to be higher than other clean cases, but there is a wide range reported from $1 \%$ to $10 \%$ depending on the type of mesh, technique, and patient population ${ }^{(3)}$. Infection of abdominal wall prostheses can have grave and costly consequences and severe impact on the patient's life due to prolonged hospitalizations and multiple re interventions, as well as very elevated social costs ${ }^{(4)}$. So, these are an incentive to explore any and all means that might reduce the incidence of mesh infection ${ }^{(5)}$. Numerous types of prosthesis have been developed to provide greater strength and lower recurrence rates, and at the same time, the risk of infection and other complications have been decreased ${ }^{\left({ }^{(6)}\right.}$. Some known risk factors for mesh infection have been reported prolonged operative time or types of mesh are predictive factors in heterogeneous series of groin hernia repairs or AWHR ${ }^{(7)}$. On the other hand, postoperative surgical site infections or concomitant intra-abdominal procedures have been related to mesh hernia repair ${ }^{(8)}$. Complete removal of the infected mesh (CMR) has been recommended if the infection cannot be resolved by conservative measures and/or antibiotic therapy or partial removal of mesh ${ }^{(9)}$. However, this fact generally induces a hernia recurrence and needs subsequent surgical procedures such as autologous flap reconstruction or another mesh implantation after the infection has been resolved ${ }^{(\mathbf{1 0})}$. CMR also can lead to a high 
complications rate, until $36-50 \%$, due to adhesions and high complexity during its removal (11). Therefore, salvage of the infected mesh without surgical removal would be desirable. An alternative to CMR is the partial removal of mesh (PMR). PMR means the excision of the nonintegrated mesh; although less frequently leads to failures and complications, some patients still require many reoperations for healing to take place $^{(12)}$.

\section{AIM of the WORK}

This work aimed to focus on management of infected mesh after ventral hernia repair.

\section{PATIENTS and METHODS}

Study population: this study was conducted in the general surgery Department, Al-Azhar University Hospitals (Sayed Galal and Al-Hussein Hospitals) in the period from September 2016 to March 2018. This prospective study included forty patients presented by infected mesh post ventral hernia repair. The study was approved by the Ethics Board of Al-Azhar University.

Inclusion criteria: patients with infected mesh after ventral hernia repair, patients who agreed the procedure and understand its risks, fit for surgery prosthetic infection was diagnosed when pathogenic organisms were found in the per prosthetic fluid obtained by surgical drainage or percutaneous puncture using ultrasound.

Exclusion criteria: patients with laparoscopic hernia repair, patients with inguinal and femoral hernia, minor infections such as cellulitis that could be treated with antibiotics alone, medically unfit for surgery, patients who didn't agree to the procedure and its risks, patients will be included if they agreed to be included in the study and an informed consent was taken.

\section{Preoperative evaluation:}

Ethical considerations were covered by standard pre-operative consent following proper instructions/guidelines from the
Ministry of Health (Clinical/surgical methods, biological samples/laboratory tests, etc...).

All patients were subjected to preoperative full history taking and included personal history such as name, age, sex, residence, occupation, marital status, special habits of medical importance and menstrual history for females, analysis of the main complaint chronic infections with fistulas chronically secreting a pus-like liquid. A detailed present history was taken regarding the onset, duration and course of symptoms and past history of previous operations and its postoperative events (wound infection, ileus, distention, wound dehiscence and respiratory complications), chronic diseases (as cardiac diseases, Diabetes Mellitus...etc.), drug allergy and intake and blood transfusion.

Microbiology data were collected on all patients. Additional variables of interest included postoperative surgical site infection (SSI) and history of previous surgical debridement.Our patients were subjected to preoperative clinical examination including general examination for vital signs and other systems to assess fitness for surgery and anesthesia. Local examination (Abdominal examination) was done. In most cases clinical presentation was a cutaneous fistula and if there is any other signs of hernia complications(obstruction, strangulation, ... etc.). In addition, the abdomen was examined for any organomegaly or other intra-abdominal co pathology to deal with it during hernia repair.

Laboratory (Routine) investigations were done for all patients including complete blood count (CBC),PC, PT, INR, ALT, AST, Urea, Creatinine, blood sugar, and serum albumin, Culture and sensitivity for aspirated fluid from collection. also radiological investigations such as abdominal ultrasonography to exclude any intraabdominal co-pathology, CT Fistulogram, sinogram and Chest plain x-ray study in cases of previous history of smoking, bronchial asthma, or clinical signs of chest troubles, Cardiological examination and electrocardiogram and echocardiography if needed.

Patients were admitted the day before surgery except for diabetic patients who were admitted 2 days before operation for control of 
blood sugar. Patients were asked to fast for at least 8 hours prior to surgery. Colonic preparation if large bowel fistula was detected.The night before surgery, preparation of the site of the operation then hair shaving just before operation at the morning. A single dose of broad spectrum antibiotic was given with induction of anesthesia.

\section{Post-operative:}

1- All patients received postoperative antibiotics, most of the patients received pre and post-operative antibiotics.

2- All of the patients with known MRSA infection were treated with vancomycin.

3- The antibiotics were adjusted in the postoperative period, according to the results of the bacterial cultures obtained from mesh samples or fluids taken during the operation, or according to the patient's clinical progression.

4- Patients were encouraged for immediate ambulation, prophylactic antibiotics, postoperative analgesics were administered. Post-operative photographs were taken.

5- Patients were usually discharged on the 2nd or 3rd day post-operative, viability of the flaps and umbilicus was checked before discharged, they were discharged on prophylactic antibiotics, analgesics with advice to Avoid straining; strenuous physical activity, minimize their physical effort and daily dressing for the wounds.

6- Abdominal binder was used for 1 month day and night,but in the second month Abdominal binder was used during daytime only.

\section{Follow up and assessments}

Follow up was done in the outpatient clinic, Drain were usually removed within a period of one week or when it drains less than $30 \mathrm{~mL}$ per day and there is no evidence of collection. Sutures were usually removed after 2 or 3 weeks according to condition of the wound. All patients were followed for up to 6 months. The follow up sessions were at two weeks, one month, two months, four months, six months after discharge from hospital.

Each follow up session included:
- History taking: for any postoperative pain, postoperative complications, seroma formation, types and duration of the patient's activities after surgery and any complain related to the surgery.

- Clinical assessment: for any signs of recurrence, any seroma collected, and tenderness at the site of the mesh.

- Laboratory and radiological assessment in selected cases.

\section{Statistical Analysis}

The statistical analysis was done using the statistical package of services solutions (SPSS; SPSS Inc., Chicago, IL, USA) software, version 21.0. Exploratory analysis and testing of continuous data for normality of distribution is done using Kolmogorov-Smirnov statistic and Shapiro- Wilk statistic. Continuous data with normal distribution are expressed in terms of (mean \pm standard deviation) while nonparametric data are expressed as median and range, categorical data presented in the form of proportion and number. $\mathrm{P}$ value considered to be important $<0.05$.

\section{RESULTS}

Table 1: patient's demographics

\begin{tabular}{|l|c|}
\hline \multicolumn{1}{|c|}{ Variable } & $\begin{array}{c}\text { Mean } \pm \text { SD / } \\
\text { frequency (\%) }\end{array}$ \\
\hline Age & $57,2 \pm 12,7$ \\
\hline Sex(male/female) & $16 / 24$ \\
\hline Smoking & $14(35 \%)$ \\
\hline Diabetes mellitus & $4(10 \%)$ \\
\hline BMI & $29,3 \pm 6,2$ \\
\hline
\end{tabular}

Table 2: microbiology of mesh infection

\begin{tabular}{|l|c|}
\hline \multicolumn{1}{|c|}{ Bacterial agent } & $\begin{array}{c}\text { Frequency } \\
(\mathbf{\%})\end{array}$ \\
\hline MRSA only & $26(65 \%)$ \\
\hline $\begin{array}{l}\text { MRSA / MSSA / K pneumoniae / P } \\
\text { mirabilis }\end{array}$ & $4(10 \%)$ \\
\hline Non MRSA infection & $10(25 \%)$ \\
\hline
\end{tabular}

Table 3: pre-operative antibiotics usage

\begin{tabular}{|l|c|c|}
\hline \multicolumn{1}{|c|}{ Antibiotic regimen } & $\begin{array}{c}\mathbf{N} \\
\text { patients }\end{array}$ & $\begin{array}{c}\text { Period } \\
\text { (days) }\end{array}$ \\
\hline Vancomycin & 18 & 10 \\
\hline Vancomycin/Meropenem & 2 & 6 \\
\hline Methicillin & 2 & 5 \\
\hline None & 18 & \\
\hline
\end{tabular}

Table 4: onset of symptoms

\begin{tabular}{|l|l|}
\hline $1-3$ month & Seroma \\
\hline $3-20$ months & Sinus \\
\hline \multirow{2}{*}{$1-2$ years } & Small bowel \\
\cline { 2 - 2 } & Large bowel \\
\hline
\end{tabular}


Table 5: characteristics of mesh infection

\begin{tabular}{|l|l|l|}
\hline \multicolumn{2}{|c|}{ Variable } & \multicolumn{2}{c|}{ Frequency(\%) } \\
\hline Presentation \\
\hline Chronic sinus & \multicolumn{2}{c|}{$30(75 \%)$} \\
\hline Infected Seroma & \multicolumn{2}{|c|}{$8(20 \%)$} \\
\hline \multirow{2}{*}{ Entericfistula } & LB & $1(2.5 \%)$ \\
\cline { 2 - 3 } & SB & $1(2.5 \%)$ \\
\hline
\end{tabular}

Table 6: surgical management of infected mesh

\begin{tabular}{|l|c|c|c|}
\hline & $\begin{array}{c}\text { Deroofing } \\
\text { and } \\
\text { drainage }\end{array}$ & $\begin{array}{c}\text { Partial } \\
\text { mesh } \\
\text { removal } \\
\text { (PMR) }\end{array}$ & $\begin{array}{c}\text { Total } \\
\text { mesh } \\
\text { removal } \\
\text { (TMR) }\end{array}$ \\
\hline Sinus & 0 & 30 & 0 \\
\hline Seroma & 8 & 0 & 0 \\
\hline $\begin{array}{l}\text { Enteric } \\
\text { fistula }\end{array}$ & 0 & 0 & 2 \\
\hline
\end{tabular}

Table 7: possible causes for persistence of infection

\begin{tabular}{|l|c|}
\hline \multicolumn{1}{|c|}{ Variable } & Frequency(\%) \\
\hline Unincorporated polypropylene & $18(45 \%)$ \\
\hline Polypropylene sutures & $10(25 \%)$ \\
\hline Multifilament sutures & $4(10 \%)$ \\
\hline $\begin{array}{l}\text { Multifilament and polypropylene } \\
\text { sutures }\end{array}$ & $4(10 \%)$ \\
\hline $\begin{array}{l}\text { Mesh over mesh and } \\
\text { polypropylene sutures }\end{array}$ & $2(5 \%)$ \\
\hline Bowel erosion by mesh & $2(5 \%)$ \\
\hline
\end{tabular}

Table 8: post-operative antibiotics usage

\begin{tabular}{|l|c|c|}
\hline \multicolumn{1}{|c|}{ Antibiotic regimen } & $\begin{array}{c}\text { N } \\
\text { patients }\end{array}$ & $\begin{array}{c}\text { Period } \\
\text { (days) }\end{array}$ \\
\hline Vancomycin & 15 & $\begin{array}{c}4- \\
21(\text { mean10) }\end{array}$ \\
\hline $\begin{array}{l}\text { Vancomycin/Piperacillin- } \\
\text { Tazobactam }\end{array}$ & 5 & 15 \\
\hline Vancomycin/Ciprofloxacin & 2 & 11 \\
\hline Vancomycin/Meropenem & 2 & 14 \\
\hline Methicillin/Ciprofloxacin & 2 & 7 \\
\hline Cefazolin/Cephalexin & 2 & 7 \\
\hline
\end{tabular}

Table 9: short and long-term outcomes

\begin{tabular}{|l|c|}
\hline \multicolumn{1}{|c|}{ Variable } & Frequency(\%) \\
\hline No complication & $32(80 \%)$ \\
\hline Major wound infection & $5(12.5 \%)$ \\
\hline Minor wound infection & $2(5 \%)$ \\
\hline Mortality & $1(2.5 \%)$ \\
\hline
\end{tabular}

Table 10: explanted mesh location

\begin{tabular}{|c|c|}
\hline Variable & Frequency(\%) \\
\hline Onlay & $30(68,2)$ \\
\hline Pre-peritoneal & $6(13,6)$ \\
\hline Retro-muscular & $4(9.1)$ \\
\hline
\end{tabular}

\section{DISCUSSION}

Abdominal wall hernia represents a major clinical burden to the workforce of general and plastic surgeons. Although outcomes of surgical repair have improved, several key issues remain unanswered. A welldefined hernia in a clean setting can be addressed effectively with a retro rectus or underlay synthetic mesh. How to repair a hernia in a contaminated or infected setting with significant loss of domain, however, remains a challenge ${ }^{(13)}$. Mesh infection is a serious postoperative complication after prosthetic hernia surgery and removal of the mesh is still the usual treatment. However, mesh removal generally results in hernia recurrence, necessitating subsequent surgical procedures such as autologous tissue repair or biological mesh placement.

The rate of infectious complications is influenced considerably by both surgeryrelated factors such as operation time, size of the defect and the type of prosthesis used, as well as by underlying medical co-morbidities such as chronic obstructive pulmonary disease, steroid use, diabetes, smoking and obesity ${ }^{\left({ }^{(14)}\right.}$.

Patients with infected mesh represent a particularly difficult group to treat due to multiple prior operations, obliterated anatomical planes, loss of domain, and presence of infected foreign object that frequently necessitates urgent intervention ${ }^{(15)}$.

Seroma appeared within one to three months, whilst sinus appeared within three to twenty months, moreover, within one to two years small bowel (SB) and large bowel (LB) appeared. In compliance with our results, Patient-related factors, including diabetes, morbid obesity, nutritional deficit, smoking, steroid therapy and renal disease, have been associated with increased risk of developing a mesh-related. This is as a result of reduced perfusion of the skin and subcutaneous tissue and immunosuppression linked to these comorbidities ${ }^{(16)}$. The obese population is known to have diminished blood supply to the tissue involved with hernia repairs and thus have impaired wound healing, putting them at particularly high risk of infection. The reduced oxygen tension inherent to the obese patient decreases the ability of polymorphonuclear leukocytes to kill bacteria and thus Weight of surgery-related infections ${ }^{\left({ }^{17}\right)}$. In hernia repairs, these obese patients often require more extensive incisions to adequately repair their defects, thus creating larger areas of "dead space" that can also lead to infection. Adipose cells in the obese patient have been documented to be in an inflammatory state. This 
inflammatory state has been linked to many comorbid illnesses and may also explain the increased infection rate in the obese population ${ }^{(18)}$. A review of the literature on mesh infection reveals that at least an attempt at mesh salvage with conservative treatment was made in each case initially, and some were in fact successful (14). No group comments that they immediately explanted the mesh upon diagnosis of infection. Most ultimately came to the realization that the infected/exposed mesh was not salvageable.

Prosthetic mesh is most commonly used as part of abdominal wall hernia repairs due to its success in reducing hernia recurrence. However, it is associated with a greater risk of infection which traditionally would have necessitated a return trip to the operating theatre for the patient, removal of the infected mesh and probable recurrence of the hernia. Due to the potential difficulty in mesh removal and the associated potential for hernia recurrence ${ }^{(19)}$. In accordance with our study, Berrevoet et al. ${ }^{(20)}$ reported that on early mesh infection only, within the first few weeks after surgery. It appears that mesh infection occurs during integration of the mesh, which is confirmed by a lack of integration signs in infected meshes. Although it is possible that the number of blood vessels in fibrotic tissue decreases, thereby reducing blood supply to the mesh and obstructing granulocyte access, this hypothesis cannot be confirmed by our series, as large pore meshes did not lower the overall infection rate. Moreover, Klinge et al. reported that, it was striking that the only meshes that had to be completely or partially removed because of ongoing infection and the lack of granulation tissue covering the mesh were multifilament polyester meshes. It is known that multifilament meshes are more prone to persistent infection than monofilament meshes. Subsequently, biologic mesh has been identified as being of value in certain abdominal wall hernia repairs due to their ability to resist infection, but because of the high cost of these meshes, surgeons need to be selective in their use ${ }^{(\mathbf{1 6})}$.Parallel with our results, antibiotics are an important component of this mesh salvage plan. The duration of antibiotics is not clearly defined. However, the antimicrobial agent selected should be directed at the more commonly encountered bacteria, Staphylococcus aureus and Streptococcus epidermiditis. Another study have even described successful salvage of composite mesh in the presence of methicillin resistant $S$. aureus (MRSA). In the case of a documented MRSA infection, usually longer courses of antibiotics are warranted, as this particular infection can be more difficult to eradicate ${ }^{(21)}$.The management of infected mesh might differ according to the type of mesh used. Specifically, it is suggested that infection of polyester or polypropylene mesh might be managed with drainage and antimicrobial agents only, whereas the infected mesh should be surgically removed in cases of infection involving expanded polytetrafluoroethylene mesh ${ }^{(22)}$. This may be attributed to the fact that a PP mesh becomes incorporated into the anterior abdominal wall with neovascularization within 2 weeks of implantation, allowing leukocytes and macrophages to gain access to the local microenvironment. Local management includes removal of skin sutures, opening of wound and drainage of pus, irrigation with saline/povidone-iodine and gentle debridement of the wound (23). Moreover, Surgical management includes mesh explanation which consists of opening the prior incision and extirpating the mesh, sutures and tacks, with closure of the fascia, if possible. More recently, there has been a trend toward mesh salvage, as explanation is plagued by hernia recurrence, loss of domain and risk of enterotomy or enterocutaneous fistula formation to solve this problem. Rectus abdominis myofascial flap closure, known as 'separation of parts' hernia repair, can be done to reduce the hernia recurrence rates ${ }^{(24)}$.

\section{Limitations of this study}

The observational nature and the small study size are important limitations of this work. Another limitation of this study was the lack of the data concerning the type of mesh and their position employed during initial surgery. However, the results of this study may provide the foundation for the understanding, care, and management of these patients. Moreover, our analysis is weakened by biased recollections. However, this study recognizes the importance of clinical examination and the surgeon's judgments regarding treatment options for these often-complex patients.

Each patient must be evaluated and treated on a case by- case basis, rather than receive therapy based on generalizations. Patience, watchful waiting, and attention to 
detail can save these patients considerable potential pain, morbidity, and delay in their recovery.

\section{CONCLUSION}

Management of Infected Mesh Following Ventral Hernia Repair presents a number of unique challenges for the treating surgeon. With little clear evidence in the literature to support a single optimal approach, clinical judgment is important. Mesh salvage is possible in a variety of settings and mesh types, usually requiring a multimodal approach, and should be attempted in most cases. As with many surgical complications, prevention is crucial. Optimization of patient comorbidities, patient selection, preoperative management, operative approach, and meticulous technique all play an important role in the development of, and therefore the prevention of, mesh infection. Research of best practices in the surgical technique, preoperative care, and mesh materials is ongoing, and much remains to be learned on prevention and management of this complex and potentially devastating complication.

\section{REFERENCES}

1. Poulose BK, Shelton J, Phillips S et al. (2012): Epidemiology and cost of ventral hernia repair: making the case for hernia research. Hernia, 16:179-183.

2. Chung L, Tse GH and O'Dwyer PJ (2014): Outcome of patients with chronic mesh infection following abdominal wall hernia repair. Hernia, 18:701-704.

3. Ousley J, Baucom RB, Stewart MK et al. (2015): Previous methicillin-resistant Staphylococcus aureus infection independent of body site increases odds of surgical site infection after ventral hernia repair. J. Am. Coll. Surg.,221:470-477.

4. Liang MK, Li LT, Nguyen MT et al. (2014): Abdominal reoperation and mesh explantation following open ventral hernia repair with mesh. Am.J. Surg.,208: 670676.

5. Mazaki T, Mado $\mathrm{K}$, Masuda $\mathrm{H}$ et al. (2014): A randomized trial of antibiotic prophylaxis for the prevention of surgical site infection after open mesh-plug hernia repair. Am. J. Surg.,207:476-484.
6. Majumder A and Novitsky YW (2015): Antibiotic Coating of Hernia Meshes: The Next Step Toward Preventing Mesh Infection. Surg. Technol. Int.,7:27-41.

7. Wiegering A, Sinha B, Spor L et al. (2014): Gentamicin for prevention of intraoperative mesh contamination: demonstration of high bactericide effect (in vitro) and low systemic bioavailability (in vivo). Hernia, 18:691-700.

8. Reslinski A, Dąbrowiecki $S$ and Glowacka K (2015): The impact of diclofenac and ibuprofen on biofilm formation on the surface of polypropylene mesh. Hernia, 19: 179-185.

9. Jezupors A and Mihelsons M (2006): The analysis of infection after polypropylene mesh repair of abdominal wall hernia. World J. Surg., 30:2270-2278.

10. Fawole AS, Chaparala RPC and Ambrose NS (2006): Fate of the inguinal hernia following removal of infected prosthetic mesh. Hernia, 10:58-61.

11. Sabbagh $C$, Verhaeghe $P$, Brehant $O$ et al. (2012): Partial removal of infected parietal meshes is a safe procedure. Hernia, 16: 445-449.

12. Tolino MJ, Tripoloni DE, Ratto $\mathrm{R}$ et al. (2009): Infections associated with prosthetic repairs of abdominal wall hernias: pathology, management and results. Hernia, 13:631-637.

13. Clay L, Stark B, Gunnarsson U et al. (2018): Full-thickness skin graft vs. synthetic mesh in the repair of giant incisional hernia: a randomized controlled multicenter study. Hernia, 22(2):325-332.

14. Munegato $G$ and Brandolese $R$ (2001): Respiratory physiopathology in surgical repair for large incisional hernia of the abdominal wall. J. Am. Coll. Surg., 192: 298-304.

15. Elfaki A, Gkorila A, Khatib $M$ et al. (2017): Infection of PTFE mesh 15 years following pedicled TRAM flap breast reconstruction: mechanism and aetiology. The Annals of the Royal College of Surgeons of England, 100(1):18-21.

16. Meagher $\mathrm{H}$, Moloney MC and Grace PA (2015): Conservative management of mesh-site infection in hernia repair surgery: a case series. Hernia, 19(2):231-237. 
17. Qadan M, Akca O, Mahid SS et al. (2009): Perioperative supplemental oxygen therapy and surgical site infection: a metaanalysis of randomized controlled trials. Archives of Surgery, 144(4):359-366.

18. Pessaux $P$, Msika $S$, Atalla $D$ et al. (2003): Risk factors for postoperative infectious complications in noncolorectal abdominal surgery: a multivariate analysis based on a prospective multicenter study of 4718 patients. Archives of Surgery, 138(3):314-324.

19. Trunzo JA, Ponsky JL, Jin J et al. (2009): A novel approach for salvaging infected prosthetic mesh after ventral hernia repair. Hernia, 13(5):545-552.

20. Berrevoet F, Vanlander AU, SainzBarriga M et al. (2013): Infected large pore meshes may be salvaged by topical negative pressure therapy. Hernia, 17(1):67-73.
21. Kercher KW, Park A, Matthews BD et al. (2002): Laparoscopic adrenalectomy for pheochromocytoma. Surgical Endoscopy and Other Interventional Techniques, 16(1):100-102.

22. Luijendijk RW, Hop WC, Van Den Tol MP et al. (2000): A comparison of suture repair with mesh repair for incisional hernia. New England Journal of Medicine,343(6):392-400.

23. Narkhede R, Shah NM, Dalal PR et al. (2015): Postoperative mesh infection still a concern in laparoscopic era. Indian Journal of Surgery,77(4):322-328.

24. Sanchez VM, Abi-Haidar YE and Itani KM (2011): Mesh infection in ventral incisional hernia repair: incidence, contributing factors, and treatment. Surgical Infections, 12(3):205-210. 